\title{
Inversion symmetry breaking in cold atomic spin patterns
}

\author{
Ivor Krešić $^{1,2}$, Gordon Robb ${ }^{1}$, Guillaume Labeyrie ${ }^{3}$, Gian-Luca Oppo ${ }^{1}$, Robin Kaiser ${ }^{3}$, Thorsten \\ Ackemann ${ }^{1}$ \\ 1. SUPA and Department of Physics, University of Strathclyde, 107 Rottenrow East, Glasgow G4 ONG, UK \\ 2. Institute of Physics, Bijenička c. 46, 10000 Zagreb, Croatia \\ 3. Université Côte d'Azur, CNRS, Institut de Physique de Nice, 06560 Valbonne, France
}

Laser driven cold atoms in front of a retro-reflecting mirror exhibit self-organization above pump threshold. It was recently demonstrated how this system can be used to mimic a transverse lattice of interacting spins (see Fig. 1) [1]. The spontaneously formed lattice breaks both translational and rotational symmetries of the initial homogeneous spin state. By applying a constant longitudinal magnetic field of $\left|B_{z}\right| \sim 100 \mathrm{mG}$ a switching between antiferromagnetic square and ferri-magnetic hexagonal pattern ordering can be induced. We study the coupling between this magnetic field and modulations in the atomic magnetization.

The modeling of atoms as a spin-1 medium results in a set of differential equations for both the populations and coherences of the ground state magnetic sublevels. The physics of self-organization is seen more clearly by expanding the atomic density matrix via the irreducible tensor expansion. When only a longitudinal magnetic field is present, the relevant degrees of freedom are the longitudinal spin dipole and the quadrupoles representing longitudinal alignment and $|\Delta m|=2$ ground state quantum coherence.

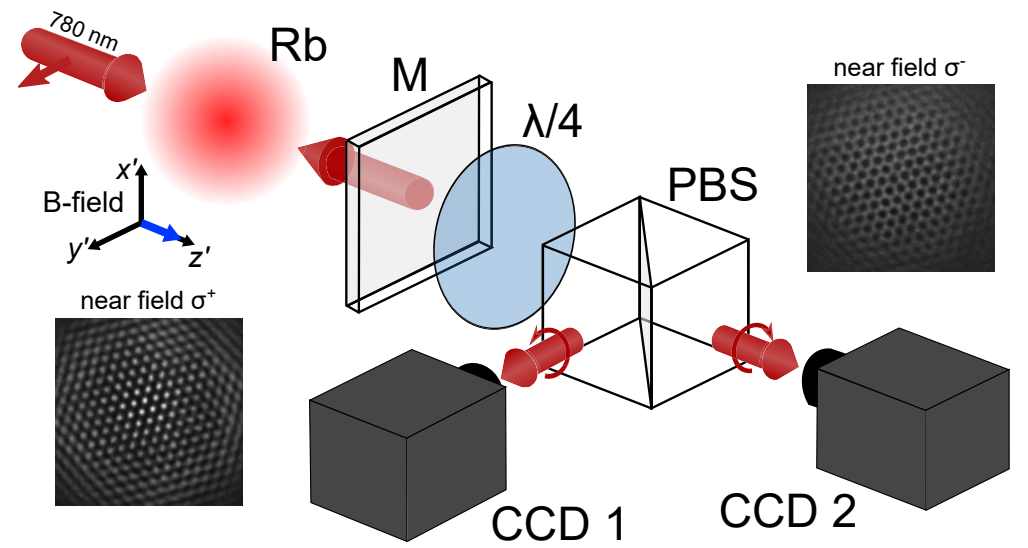

\begin{abstract}
Fig. 1 Simplified schematics of the experimental setup, adapted from [1]. A linearly polarized pump beam is reflected from a feedback mirror to drive the spin self-organization in the atomic cloud. A small transmitted part of the beam is used for polarization selective NF and FF imaging. Inversion symmetry is broken by applying a small longitudinal magnetic field (Bfield), resulting in formation of hexagons and honeycombs in the $\sigma$ polarization channels (inset: $\mathrm{NF}$ data). Rb - cold cloud of ${ }^{87} \mathrm{Rb}, \mathrm{M}$ - feedback mirror, $\lambda / 4$ - quarter-wave plate, PBS - polarizing beam splitter cube, CCD - charge-coupled device camera.
\end{abstract}

Our experimentally measured variation of pattern thresholds and symmetries with $B_{z}$ is compared to the results of both numerical calculations in the full model and analytical calculations in a reduced model allowing for perturbations only in the spin dipole [2]. The reduced model shows that inversion symmetry of the system is broken at small $\left|B_{z}\right|$ by nonlinear Faraday rotation caused by coupling of the homogeneous parts of light induced quantum coherence and spin dipole. A complex Ginzburg-Landau equation governing the wave mixing of spin roll states is derived. At large $\left|B_{z}\right|$ the behavior cannot be accounted for by the reduced model, leading to the conclusion that perturbations in magnetic quadrupoles govern the pattern behavior in this regime.

Understanding of spontaneous light-mediated atomic ordering has potential relevance for future quantum technologies. Our study could provide a basis for further research on instabilities in driven multilevel systems with feedback, which have in the past been largely unexplored due to their complexity.

\section{References}

[1] I. Krešić, G. Labeyrie, G. R. M. Robb, G.-L. Oppo, P. M. Gomes, P. Griffin, R. Kaiser, and T. Ackemann, "Spontaneous light-mediated magnetism in cold atoms", Commun. Phys. 1, 33 (2018).

[2] I. Krešić, G. R. M. Robb, G. Labeyrie, G.-L. Oppo, R. Kaiser, and T. Ackemann, "Inversion symmetry breaking in spin patterns by a weak magnetic field", (in preparation). 